\title{
Topological phases of higher Chern numbers in Kitaev-Heisenberg ferromagnet with further-neighbor interactions
}

\author{
Moumita Deb ${ }^{1, *}$ and Asim Kumar Ghosh ${ }^{1, \dagger}$ \\ ${ }^{1}$ Department of Physics, Jadavpur University, 188 Raja Subodh Chandra Mallik Road, Kolkata 700032, India
}

\begin{abstract}
Emergence of multiple topological phases with a series of Chern numbers, $\pm 1, \mp 1, \pm 2, \mp 2$, \pm 3 and $\mp 4$, is found in a ferromagnetic Kitaev-Heisenberg-spin-anisotropic model on honeycomb lattice with next-next-nearest-neighbor interactions in the presence of an external magnetic field. Magnon Chern insulating dispersions of this two-band model are studied by using linear spin-wave theory formulated on the exact ferromagnetic ground state. Magnon edge states are obtained for the respective topological phases along with density of states. A topological phase diagram of this model is presented. Behavior of thermal Hall conductivity for those phases is studied. Sharp jumps of thermal hall conductivity is noted near the vicinity of phase transition points. Topological phases of the Kitaev spin-liquid compounds, $\alpha-\mathrm{RuCl}_{3}, \mathrm{X}_{2} \mathrm{IrO}_{3}, \mathrm{X}=(\mathrm{Na}, \mathrm{Li})$ and $\mathrm{CrY}_{3}, \mathrm{Y}=(\mathrm{Cl}, \mathrm{Br}, \mathrm{I})$ are characterized based on this theoretical findings.
\end{abstract}

PACS numbers:

\section{INTRODUCTION}

Investigation on topological properties of matter experiences mammoth growth in the recent times. The lion's share of those studies involves in the fermionic systems those are described in terms of tight-binding models on various lattice structures ${ }^{1}$. A major portion of those findings deals with the characterization of topological phases of multi-band models based on the values of several topological invariants those are dependent on the dimension and symmetry of the systems. For the two-dimensional systems, topological phases are characterized in terms of topological invariant, Chern number $(C)$, for every distinct energy bands when the time reversal symmetry (TRS) is broken ${ }^{2}$. Nontrivial topological phases are identified when all the $C$ s of the bulk energy-bands are not simultaneously zero, the resulting systems are collectively known as topological insulators (TI). Nonzero values of $C$ is further associated with the presence of chiral edge states connecting the respective bulk bands in accordance to the 'bulk-boundary-correspondence' (BBC) rule ${ }^{3-5}$.

However, study of similar types of topological phases in the bosonic systems described in terms of Heisenberg models has been started in the more recent time. The number of investigated bosonic systems is therefore less in comparison to that of the fermionic systems. Search of topological phase in the ferromagnetic (FM) systems begins with the finding of thermal Hall conductance in the Mott insulator, $\mathrm{Lu}_{2} \mathrm{~V}_{2} \mathrm{O}_{7}{ }^{6,7}$. In this compound, spin$1 / 2 \mathrm{~V}^{4+}$ ions form a three-dimensional pyrochlore lattice which is essentially a stacking of alternating kagomé and triangular layers. Likewise, strong evidence of topological magnon insulating (TMI) phase is detected in another kagomé ferromagnet, $\mathrm{Cu}[1,3$-benzenedicarboxylate (bdc)] in the presence of external magnetic field ${ }^{8}$.

Nontrivial topological phases for $\mathrm{Lu}_{2} \mathrm{~V}_{2} \mathrm{O}_{7}$ are explained theoretically by considering a minimal model of spin-1/2 FM Heisenberg model on a kagomé lattice in the presence of antisymmetric Dzyaloshinskii-Moriya (DM) interaction $^{9,10}$, where this term acts as a vector potential. In this case, the resulting three magnon bands are characterized by $C=1,0,-1$, where the Zeeman term associated with the magnetic field breaks TRS ${ }^{11,12}$. The system further exhibits topological phase transition (TPT) at the point having zero DM strength. Another TMI phase with $C= \pm 1$ is obtained later in magnon dispersion of two-band honeycomb ferromagnet with DM interaction ${ }^{13,14}$. For the antiferromagnetic (AFM) case, Heisenberg ShastrySutherland model with DM interaction exhibits two distinct TMI phases with specific values of $C=-2,0,2$ and $C=-1,0,1$, obtained for three bosonic triplon dispersion bands under longitudinal and cross magnetic fields, respectively ${ }^{15,16}$.

Previous studies reveal that values of $C \mathrm{~s}$ for all of the FM bosonic systems are not more than one. However, emergence of topological phases with higher values of $C$ in fermionic systems is found before either invoking distant-neighbor interactions ${ }^{17}$ or irradiating with circularly polarized light ${ }^{18}$. Therefore, search of other bosonic models those are capable to exhibit new topological phases as well as phases with higher values of $C$ continues in those directions. Recently, both AFM and FM models consisting of nearest-neighbor (NN) Kitaev ${ }^{19}$, Heisenberg and symmetric spin-anisotropic interactions (KHSA) are introduced those are found to exhibit TMI phases on the honeycomb lattice in the presence of magnetic field ${ }^{20,21}$. The FM KHSA system hosts the TMI phase with $C= \pm 1^{21}$. Again, value of $C$ is not more than one which is so far true for all bosonic lattice models.

In this study, we report the emergence of a variety TMI phases with higher $C$ in FM KHSA model on honeycomb lattice in the presence of next-next-nearestneighbor (NNNN) interactions in addition to the NN terms. Values of $C$ s for those additional phases in this two-band model are $\pm 2, \mp 2, \pm 3$, and $\mp 4$. Higher values of $C$ are hereby noticed for the first time on this two-band bosonic lattice model. However, additional next-nearestneighbor (NNN) KHSA interactions fail to yield such new topological phases. 
Interestingly, existence of both NN and NNNN KHSA interactions is predicted before in $\alpha-\mathrm{RuCl}_{3}$ by combined $a b$ initio and strong-coupling approaches, where spin$1 / 2 \mathrm{Ru}^{3+}$ ions constitute the honeycomb structure ${ }^{22}$. Both density functional theory and nonperturbative exact diagonalization methods also support the presence of NNNN interactions in $\alpha-\mathrm{RuCl}_{3}{ }^{23,24}$. Values of all the interaction strengths have been determined. Besides the Kitaev spin liquid ground state, $\alpha-\mathrm{RuCl}_{3}$ is recently found to demonstrate the half-quantized thermal Hall effect $^{25}$. Meanwhile, emergence of multiple TMI phases with higher values of $C$ for the model based on this compound turns the system more attractive.

However, the number of magnetic compounds those properties are explained in terms of various KHSA models is not less. KHSA Hamiltonian has been suggested for the Kitaev spin-liquid compounds, $\mathrm{X}_{2} \mathrm{IrO}_{3}, \mathrm{X}=(\mathrm{Na}, \mathrm{Li})$, based on the results of both $a b$ initio and perturbative calculations, where the spin- $1 / 2 \mathrm{Ir}^{4+}$ ions form honeycomb structure 22,26 . Recently, results of angle-dependent FM resonance (FMR) experiments reveal the presence of FM KHSA interactions between NN spin-3/2 $\mathrm{Cr}^{3+}$ ions within all chromium halides, $\mathrm{CrY}_{3}, \mathrm{Y}=(\mathrm{Cl}, \mathrm{Br}, \mathrm{I})$, where $\mathrm{Cr}^{3+}$ ions are arranged in a honeycomb lattice ${ }^{27}$.

The linear spin-wave theory (LSWT) of the FM KHSA model on the honeycomb lattice is formulated in the subsequent sections. LSWT is valid for spin-1/2 but more accurate for higher values of spin moment. Therefore, TMI phases for all the above compounds can be characterized with specific values of $C$ along with transition between them. Evidence of chiral edge states supports the values of $C$ of those TMI phases. Value of the thermal Hall conductivity (THC) is obtained to study the transition between those TMI phases. Article is arranged in the following manner. Section II describes the model along with the determination of classical ground state. LSWT is formulated in Sec III, where analytic expressions of eigenvalues are given. Topological phases have been characterized in Sec IV while Sec V contains discussion on the results.

\section{KITAEV-HEISENBERG MAGNET WITH SPIN-ANISOTROPIC INTERACTIONS ON HONEYCOMB LATTICE}

The model comprises Kitaev, Heisenberg and spinanisotropic interactions on the NN as well as NNNN bonds in the presence of an external magnetic field. The latter two interactions depend on the choice of three dif-

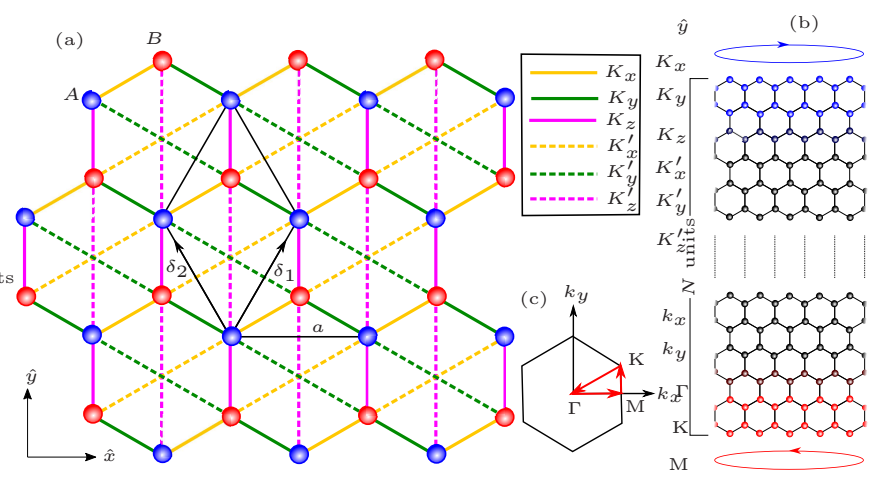

FIG. 1: (color online) (a) Geometrical view of the model with NN and NNNN interactions on the honeycomb lattice, (b) geometry of the lattice used for edge state calculation, upper and lower edges are drawn in blue and red colors, respectively, (c) the first Brillouin zone showing the high-symmetry points, $\Gamma, \mathrm{M}$ and $\mathrm{K}$.

ferent links. The Hamiltonian is written as

$$
\begin{aligned}
\mathcal{H} & =J \sum_{\langle i j\rangle} \boldsymbol{S}_{i} \cdot \boldsymbol{S}_{j}+2 \sum_{\langle i j\rangle_{\gamma}} K_{\gamma} S_{i}^{\gamma} S_{j}^{\gamma}+\sum_{\langle i j\rangle_{\gamma}} \Gamma_{\gamma}\left(S_{i}^{\alpha} S_{j}^{\beta}+S_{i}^{\beta} S_{j}^{\alpha}\right) \\
& +J^{\prime} \sum_{\langle\langle\langle i j\rangle\rangle\rangle} \boldsymbol{S}_{i} \cdot \boldsymbol{S}_{j}+\underset{\langle\langle\langle i j\rangle\rangle\rangle_{\gamma}}{2} K_{\gamma}^{\prime} S_{i}^{\gamma} S_{j}^{\gamma}+\sum_{\langle\langle\langle i j\rangle\rangle\rangle_{\gamma}} \Gamma_{\gamma}^{\prime}\left(S_{i}^{\alpha} S_{j}^{\beta}+S_{i}^{\beta} S_{j}^{\alpha}\right) \\
& -\boldsymbol{h} \cdot \sum_{i} \boldsymbol{S}_{i} .
\end{aligned}
$$

Here, $J\left(J^{\prime}\right), K_{\gamma}\left(K_{\gamma}^{\prime}\right)$ and $\Gamma_{\gamma}\left(\Gamma_{\gamma}^{\prime}\right)$ are the Heisenberg, Kitaev and spin-anisotropic interaction strengths respectively for the NN (NNNN) bonds. $\gamma=x, y, z$, which denotes three different links of the honeycomb lattice. $\boldsymbol{h}=g \mu_{\mathrm{B}} \boldsymbol{H}$, where $\boldsymbol{H}$ is the strength of magnetic field. $S_{i}^{\alpha}$ is the $\alpha$-th component of spin operator, $\boldsymbol{S}_{i}$, at the $i$-th site, where $\alpha=x, y, z$. Summations over NN and NNNN bonds are denoted by the indices $\langle\cdot\rangle$ and $\langle\langle\langle\cdot\rangle\rangle\rangle$, respectively. Periodic boundary condition (PBC) is assumed along both $x$ and $y$ directions. Both $J_{\mathrm{S}}$ and $K \mathrm{~s}$ may assume positive (AFM) and negative (FM) values separately.

Geometrical view of this spin model is given in Fig 1(a). NN (NNNN) bonds are shown by solid (dashed) lines. $\delta_{\mathbf{1}}$ and $\delta_{\mathbf{2}}$ are the primitive vectors of non-Bravais honeycomb lattice such that the parallelogram bounded by them encloses two nonequivalent sites $A$ and $B$, those are shown by blue and red spheres, respectively.

Zero temperature phase diagram of this model in the absence of NNNN interactions $\left(J^{\prime}=0, K^{\prime}=0, \Gamma^{\prime}=0\right)$ is already studied. For $\Gamma=0$, system exhibits four spin-ordered phases, Néel, zigzag, FM, stripy and two intermediate spin-liquid phases which lie between Néelzigzag and FM-stripy phases. FM phase survives for $0.85 \pi<\phi<1.5 \pi, \phi=\arctan (K / J)$, when $h=0^{28}$. With the increase of $h$, FM phase is replaced by the spin-polarized (SP) phase which begins to spread and ultimately occupy the whole parameter space, $0<\phi \leq 2 \pi$ above the critical value $h_{c}(\phi)^{29,30}$. Value of $h_{c}$ also de- 
pends on the direction of $\boldsymbol{h}$. SP phase quickly consumes the entire zigzag and stripy phases with the increase of $h$ as they reside around the FM phase. Quantum states for FM and SP phases are even though identical but their causes are different. However, in this region, the system is topologically trivial. For $\Gamma \neq 0$, additional two spinordered phases, $120^{\circ}$ order and incommensurate spiral are found to appear in the phase diagram when $S=1 / 2$ and $h=0^{31}$. Interestingly, SP phase is topologically nontrivial since the magnon dispersion bands emerge with $C= \pm 1$ and $\mp 1$, when $h>\Gamma_{z}$ and directed toward $\hat{z}$ and [111] directions, respectively ${ }^{21}$. However, no additional topological phase is found to appear in the presence of NNN interactions.

This work reveals that the system exhibits additional four topological phases $(C= \pm 2, \mp 2, \pm 3, \mp 4)$, with the inclusion of NNNN terms, when $h>\left|\Gamma_{z}+\Gamma_{z}^{\prime}\right|$. Therefore, the SP phase hosts six different topological phases in this case when $h$ points toward $\hat{z}$. Zigzag order is observed in the compounds $\alpha-\mathrm{RuCl}_{3}$ and $\mathrm{X}_{2} \mathrm{IrO}_{3}$ below the respective Néel temperatures ${ }^{24}$ while FM order is found in $\mathrm{CrI}_{3}$ below the Curie temperature ${ }^{27}$. So, the results of LSWT on the KHSA model based on the FM ground state are applicable on those materials as long as $\boldsymbol{h}$ is nonzero.

The extend of FM ground state of this model for the honeycomb lattice can be obtained in terms of that for a single unit cell. For this purpose, a hexagon is considered that holds classical spin vector each at its six vertices. Components of the spin vectors have been expressed in terms of the polar coordinates $\theta$ and $\phi$ as $\boldsymbol{S}_{n}=S\left(\sin \theta_{n} \cos \phi_{n}, \sin \theta_{n} \sin \phi_{n}, \cos \theta_{n}\right)$, where $n=$ $1,2,3,4,5,6$. The minimum value of classical energy corresponds to the spin configuration given by $\phi_{n}=0$ and $\theta_{n}=0$ when both $J_{\mathrm{s}}$ and $K \mathrm{~s}$ are negative. In this case, classical energy of a single hexagon is given by $E_{0}=6(J+2 K) S^{2}+3\left(J^{\prime}+2 K^{\prime}\right) S^{2}-6 h S$, when $K=K_{\gamma}, K^{\prime}=K_{\gamma}^{\prime}$ and $\boldsymbol{h}$ is acting along the $\hat{z}$ direction. $E_{0}$ does not depend on the spin-anisotropic interaction due to the symmetry of the spin configuration. Therefore, in the classical ground state all the spins orient along the $\hat{z}$ direction when both $J_{\mathrm{s}}$ and $K$ s are negative which in the other words corresponds to the FM ground state. However, in general, SP phase dominates as long as $h / S \geq J+2 K+\left(J^{\prime}+2 K^{\prime}\right) / 2$. Therefore, in this whole parameter regime single magnon dispersions can be treated as exact one.

\section{MAGNON DISPERSION RELATIONS}

In order to obtain the FM magnon dispersion relations based on the classical ground state, spin operators are expressed in terms of bosonic creation $\left(b^{\dagger}\right)$ and annihilation $b$ operators through the Holstein-Primakoff transformation as

$$
S_{j}^{z}=S-b_{j}^{\dagger} b_{j}, \quad S_{j}^{+} \simeq \sqrt{2 S} b_{j}, \quad S_{j}^{-} \simeq \sqrt{2 S} b_{j}^{\dagger} .
$$

Following the LSWT, Hamiltonian (Eq.1) has been expressed in the momentum space by performing the Fourier transformation of the operators, $b_{j}=$ $\frac{1}{\sqrt{N}} \sum_{\boldsymbol{k}} b_{\boldsymbol{k}} e^{i \boldsymbol{k} \cdot \boldsymbol{R}_{j}}$.

$$
\mathcal{H}=\frac{3 N S^{2}}{2}\left[J+J^{\prime}+2\left(K+K^{\prime}\right)\right]-N h S+\frac{S}{2} \sum_{\mathbf{k}} \Psi_{\mathbf{k}}^{\dagger} \mathcal{H}_{\mathbf{k}} \Psi_{\mathbf{k}},
$$

where $N$ is the total number of sites and $\Psi_{\mathrm{k}}^{\dagger}=$ $\left[b_{A, \mathbf{k}}^{\dagger}, b_{B, \mathbf{k}}^{\dagger}, b_{A,-\mathbf{k}}, b_{B,-\mathbf{k}}\right]$. Now, $b_{A}^{\dagger}$ and $b_{B}^{\dagger}$ are the bosonic creation operators on sublattices $A$ and $B$, respectively. The estimated values of $\Gamma$ and $\Gamma^{\prime}$ for the Kitaev materials are generally very small. For $\alpha-\mathrm{RuCl}_{3}$ they are given by $\Gamma / K \approx 0.7$ and $\Gamma^{\prime} / K \approx 0.0^{22} . \Gamma / K \approx 0.013$ and 0.0 for $\mathrm{CrI}_{3}{ }^{27}$ and $\mathrm{X}_{2} \mathrm{IrO}_{3}{ }^{24}$, respectively. On the other hand, topological phases appear as soon as values of $\Gamma$ and $\Gamma^{\prime}$ are nonzero, and they are found robust against any alteration of them. Therefore, one can assume both $x$ and $y$ components of $\Gamma$ and $\Gamma^{\prime}$ are negligible for the sake of analytic convenience. Otherwise, Eq 3 includes linear and cubic terms of $b$ operators. It is worth mentioning that different TMI phases appear for different values of $J_{\mathrm{S}}$ and $K$ s and a definite phase survives as long as $h>\left|\Gamma+\Gamma^{\prime}\right|$ without depending on the individual values of $h, \Gamma$ and $\Gamma^{\prime}$. Thus by keeping only the bilinear terms, form of the $4 \times 4$ matrix, $\mathcal{H}_{\mathbf{k}}$ is given below.

$$
\mathcal{H}_{\mathbf{k}}=\left[\begin{array}{cc}
X_{\mathbf{k}} & Y_{\mathbf{k}} \\
Y_{\mathbf{k}}^{\dagger} & X_{-\mathbf{k}}^{T}
\end{array}\right], X_{\mathbf{k}}=\left[\begin{array}{ll}
a_{0} & a_{\mathbf{k}} \\
a_{\mathbf{k}}^{*} & a_{0}
\end{array}\right], Y_{\mathbf{k}}=\left[\begin{array}{cc}
0 & b_{\mathbf{k}} \\
b_{-\mathbf{k}} & 0
\end{array}\right]
$$

where,

$$
\begin{aligned}
a_{0}= & -3 J-2 K-3 J^{\prime}-2 K^{\prime}+h / S, \\
a_{\mathbf{k}}= & J+(J+K)\left(e^{-i \mathbf{k} \cdot \boldsymbol{\delta}_{1}}+e^{-i \mathbf{k} \cdot \boldsymbol{\delta}_{2}}\right) \\
& +J^{\prime} e^{-i \mathbf{k} \cdot \boldsymbol{n}_{1}}+\left(J^{\prime}+K^{\prime}\right)\left(e^{-i \mathbf{k} \cdot \boldsymbol{n}_{2}}+e^{-i \mathbf{k} \cdot \boldsymbol{n}_{3}}\right), \\
b_{\mathbf{k}}= & K\left(e^{-i \mathbf{k} \cdot \boldsymbol{\delta}_{1}}-e^{-i \mathbf{k} \cdot \boldsymbol{\delta}_{2}}\right)+K^{\prime}\left(e^{-i \mathbf{k} \cdot \boldsymbol{n}_{3}}-e^{-i \mathbf{k} \cdot \boldsymbol{n}_{2}}\right) \\
& +i \Gamma_{z}+i \Gamma_{z}^{\prime} e^{-i \mathbf{k} \cdot \boldsymbol{n}_{1}}, \text { with, } \\
\boldsymbol{\delta}_{1}= & a\left(\frac{1}{2} \hat{i}+\frac{\sqrt{3}}{2} \hat{j}\right), \boldsymbol{\delta}_{2}=a\left(-\frac{1}{2} \hat{i}+\frac{\sqrt{3}}{2} \hat{j}\right), \\
\boldsymbol{n}_{1}= & \sqrt{3} a \hat{j}, \boldsymbol{n}_{2}=a \hat{i}, \boldsymbol{n}_{3}=-a \hat{i} .
\end{aligned}
$$

Here, $a$ is the lattice distance between two adjacent $A$ or $B$ sublattice sites which is henceforth set to 1 .

According to the bosonic Bogoliubov transformation, the non-Hermitian matrix $I_{\mathrm{B}} \mathcal{H}_{\mathbf{k}}$ is diagonalized in order to obtain the eigenenvalues and eigenmodes where $I_{\mathrm{B}}=\operatorname{diag}[1,1,-1,-1]$. The characteristic equation in terms of the eigenvalue, $E_{\mathbf{k}}$, is given by $E_{\mathbf{k}}^{4}+p_{\mathbf{k}} E_{\mathbf{k}}^{2}+$ $q_{\mathbf{k}}=0$, where $p_{\mathbf{k}}=\left|b_{\mathbf{k}}\right|^{2}+\left|b_{-\mathbf{k}}\right|^{2}-2\left(a_{0}^{2}+\left|a_{\mathbf{k}}\right|^{2}\right)$ and $q_{\mathbf{k}}=\left(a_{0}^{2}-\left|a_{\mathbf{k}}\right|^{2}\right)^{2}-a_{0}^{2}\left(\left|b_{\mathbf{k}}\right|^{2}+\left|b_{-\mathbf{k}}\right|^{2}\right)+\left|b_{\mathbf{k}}\right|^{2}\left|b_{-\mathbf{k}}\right|^{2}-$ $a_{\mathbf{k}}^{2} b_{\mathbf{k}}^{*} b_{-\mathbf{k}}-a_{\mathbf{k}}^{* 2} b_{\mathbf{k}} b_{-\mathbf{k}}^{*}$. Real eigenvalues are found as long as $h>\left|\Gamma_{z}+\Gamma_{z}^{\prime}\right|$. Magnon dispersion relations are given by the positive eigenvalues of the characteristic equation, $E_{\mathbf{k}}^{ \pm}=\left[\left(-p_{\mathbf{k}} \pm \sqrt{p_{\mathbf{k}}^{2}-4 q_{\mathbf{k}}}\right) / 2\right]^{\frac{1}{2}} . E_{\mathbf{k}}^{ \pm}$with specific values of $C$ have been plotted in Fig 2 for four different TMI 

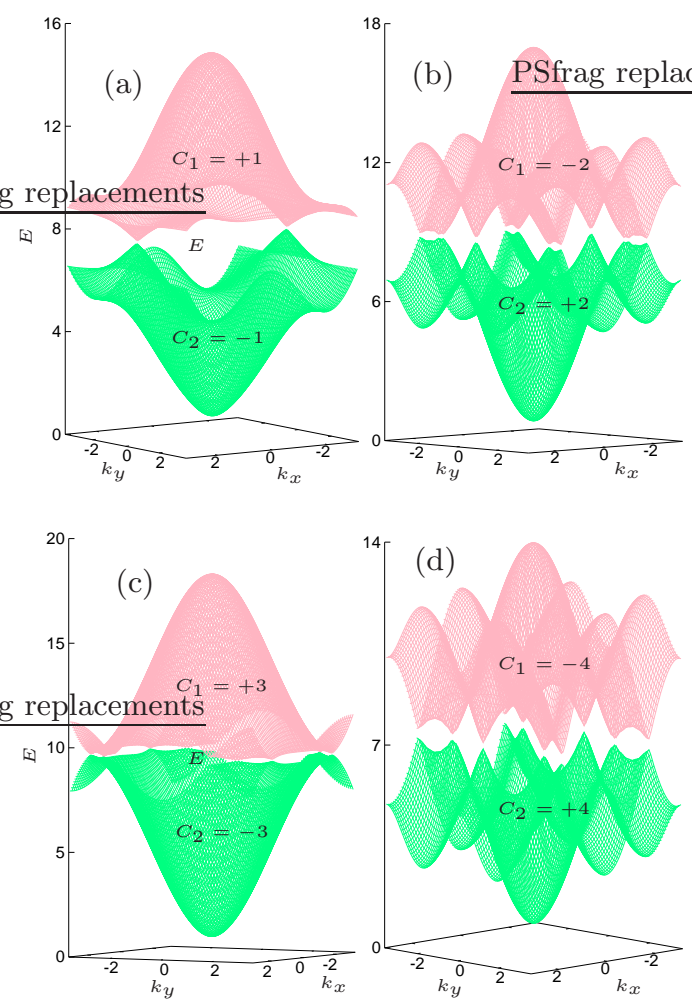

FIG. 2: (color online) Three-dimensional magnon bands. (a) $J=-1, K=-1, K^{\prime}=-1, \Gamma_{z}^{\prime}=0.5$ for $C= \pm 1$, (b) $J=-1, J^{\prime}=-1, K^{\prime}=-1, \Gamma_{z}^{\prime}=0.5$ for $C=\mp 2,(\mathrm{c})$ $J=-2, K=-0.2, J^{\prime}=-1, K^{\prime}=0.4, \Gamma_{z}^{\prime}=0.5$ for $C= \pm 3$, and (d) $J=-0.5, J^{\prime}=-1, K^{\prime}=-1.0, \Gamma_{z}^{\prime}=0.5$ for $C=\mp 4$. No value is specified for those parameters when they are zero.
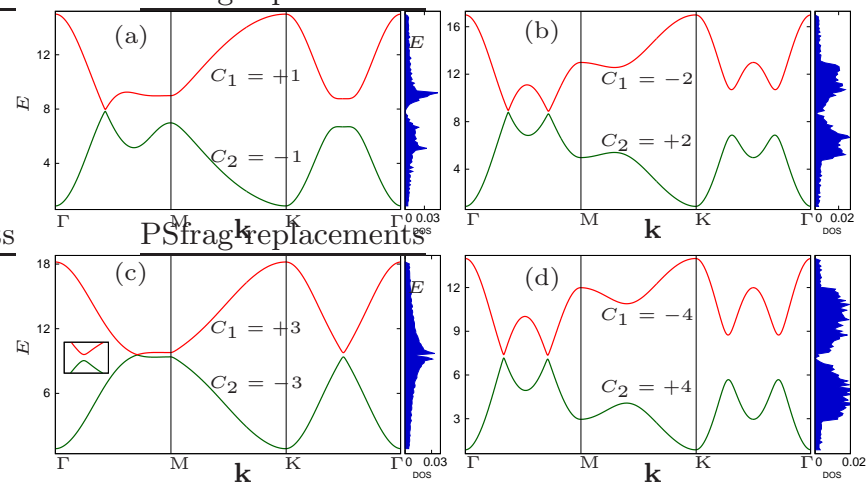

FIG. 3: (color online) Dispersion relation along the highsymmetry points of Brillouin zone. The side panel shows the density of states. Values of the parameters are the same as Fig 2 for the respective plots.

phases, (a) $C= \pm 1$, (b) $C=\mp 2$, (c) $C= \pm 3$, and (d) $C=\mp 4$.

Number of band touching points is the minimum in Fig 2 (a) where $C= \pm 1$, while it is the maximum in (d) when $C=\mp 4$. Dispersion relations along the highsymmetry points of BZ in addition to the density of states (DOS) are shown in Fig 3 for four different cases. DOS reveals the presence of true band gap in every case. The
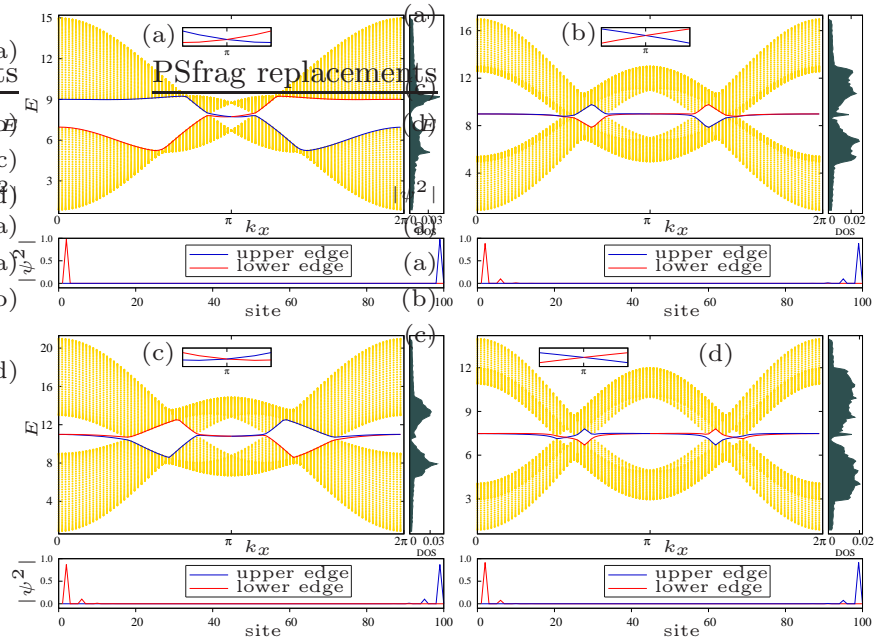

FIG. 4: (color online) Magnon dispersions of edge and bulk states in the one-dimensional BZ. Upper and lower edge modes are drawn in blue and red lines, respectively, while bulk modes are in golden points. Insets show the closer view of crossing points of edge energies around a definite value of $k_{x}$. The side panel shows the density of edge states. The lower panel indicates variation of probability density of both edge modes with respect to site number for a fixed $k_{x}$. Values of the parameters are: (a) $J=-1, K=-1, K^{\prime}=-1, \Gamma_{z}^{\prime}=0.5$ for $C= \pm 1$, (b) $J=-1, J^{\prime}=-1, K^{\prime}=-1, \Gamma_{z}^{\prime}=0.5$ for $C=\mp 2,(\mathrm{c}) J=-1, K=-1, J^{\prime}=-1, K^{\prime}=-1, \Gamma_{z}^{\prime}=0.5$ for $C= \pm 2$, and (d) $J=-0.5, J^{\prime}=-1, K^{\prime}=-1.0, \Gamma_{z}^{\prime}=0.5$ for $C=\mp 4$. No value is assigned to those parameters when they are zero.

presence of NNN interactions in this specific model only alter the diagonal elements of the Eq 4. Thus it fails to generate additional TMI phase since the values of $C$ are found technically insensitive to the values of diagonal components.

\section{CHERN NUMBERS, EDGE STATES AND THERMAL HALL CONDUCTANCE}

In order to draw the topological phase diagram, value of Chern number and edge states have been obtained throughout the parameter space of the system. Chern number of a particular band has been calculated by integrating the Berry curvature over the Brillouin zone (BZ).

$$
C=\frac{1}{2 \pi} \iint_{B Z} F(\mathbf{k}) d k_{x} d k_{y},
$$

where the Berry curvature of that band, $F(\mathbf{k})$, is expressed in terms of the corresponding Berry connection, $A_{\mu}(\mathbf{k})=\left\langle n(\mathbf{k})\left|\partial_{k_{\mu}}\right| n(\mathbf{k})\right\rangle$ as $F(\mathbf{k})=\partial_{k_{x}} A_{y}(\mathbf{k})-$ $\partial_{k_{y}} A_{x}(\mathbf{k})$, and $|n(\mathbf{k})\rangle$ is the eigenvector of that particular magnon band. Ultimately, the value of $C$ has been estimated numerically following the method proposed by Fukui and others ${ }^{32}$.

Nonzero value of $C$ implies the existence of edge states according to the $\mathrm{BBC}$ rule. Edge states correspond to the 


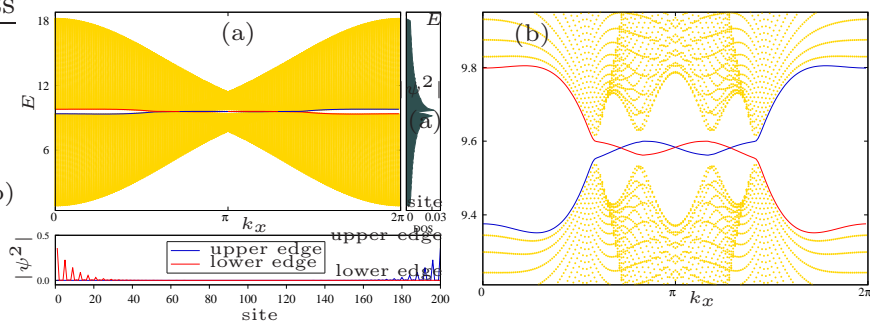

FIG. 5: (color online) (a) Bulk and edge magnon mode energies showing the existence of TMI phase with $C= \pm 3$, when $J=-2, K=-0.2, \Gamma_{z}=0.0, J^{\prime}=-1, K^{\prime}=0.4$, and $\Gamma_{z}^{\prime}=0.5$, (b) a closer view of the three crossing points around $9<E<10$.

surface property of the system. A pair of edges parallel to the $x$-axis has been created by breaking the PBC along the $y$-axis. For this purpose, a strip of honeycomb lattice is considered which has $N$ unit cells along the $\hat{y}$ and infinitely long towards the $\hat{x}$. This fact is taken into account by retaining the $\mathrm{PBC}$ along $x$ axis where $k_{x}$ is treated as a good quantum number. Subsequently, taking Fourier transform of the bosonic operators only along the $x$ direction, $2 N \times 2 N$ Hamiltonian has been obtained. Diagonalizing the Hamiltonian for $N=50$, eigenvalues and eigenvectors of the bulk as well as edge states have been obtained.

Dispersions of edge and bulk states in the onedimensional BZ for four different topological phases have been shown in Fig 4. Edge state dispersion branches for upper (blue) and lower (red) sides are drawn in different colors. For this two-band system number of crossing between those two dispersion branches is actually proportional to the absolute value of $C$ for those respective bands. TMI phase for $J=-1, K=-1, \Gamma_{z}=0, J^{\prime}=0$, $K^{\prime}=0, \Gamma_{z}^{\prime}=0.5$ corresponds to $C=\mp 1$, where the upper sign denotes the value of $C$ for upper energy band. Exchange of Chern numbers occurs when NNNN Kitaev interaction becomes equal to $-1\left(K^{\prime}=-1\right)$, leading to another TMI phase with $C= \pm 1$, which is shown in Fig 4 (a). TMI phase with $C= \pm 2$ appears when $J^{\prime}=-1$ which is followed by another TMI phase with $C=\mp 2$ when NN Kitaev interaction is made zero $(K=0)$. Those are shown in Figs 4 (c) and (b), respectively. System exhibits TMI phase with $C=\mp 4$ when $J$ is fixed at -0.5 keeping other parameters unchanged, which is shown in Fig 4 (d). Fig 5 (a) demonstrates the dispersion bands of another TMI phase with $C= \pm 3$, where the closer view of three distinct crossing points of edge states are shown in Fig 5 (b).

Phase diagram profile of the system is shown in Fig 6 for $h=1.0, \Gamma_{z}=0, \Gamma_{z}^{\prime}=0.5$ and when $J^{\prime}=K^{\prime}$. Three diagrams (a), (b) and (c) are drawn by varying $K$ and $J^{\prime}$ but keeping the values of $J$ fixed at $0.0,-0.5$ and -1.0 , respectively. Region I corresponds to $C=\mp 4$. This phase dominates when $J=0$, but begins to diminish with the decrease of $J$ and ultimately disappears at $J=-1$. Likewise, regions II, III, V and VI corresponds to $\mp 2$, $\mp 1, \pm 1$ and \pm 2 , respectively. Region III appears only when $J<0$. On the other hand, region IV is a trivial one $(C=0)$ and it is found to appear in every diagram. Another nontrivial phase with $C= \pm 3$ that does not appear in Fig 6, but is found in one of a typical situation where $J=-2, J^{\prime}=-1, K<0$ and $K^{\prime}>0$. Therefore, in total six distinct TMI phases appear in this system.

Interestingly, Chern numbers are found to exchange sign with the change of sign of $\Gamma_{z}+\Gamma_{z}^{\prime}$, when $h>\left|\Gamma_{z}+\Gamma_{z}^{\prime}\right|$. This behavior can be understood in terms of TPT of the system across the boundary line $L, \Gamma_{z}+\Gamma_{z}^{\prime}=0$, which is shown in Fig 7. For $J=K=J^{\prime}=K^{\prime}=-1$, the system undergoes a transition from phase \pm 2 in region $A$ to phase $\mp 2$ in region $B$, across the line $L$. Similarly, for $J=K=-1$ and $J^{\prime}=K^{\prime}=-0.5$, the system exhibits phase \pm 1 in region $A$ and $\mp 1$ in region $B$. TMI phase does not survive over the line $L$, since no band-gap is found there. However, similar kind of transition is not observed for the phases \pm 3 and $\mp 4$ for obvious reason.

THC, $\kappa_{x y}$, of the system can be expressed in terms of $F(\mathbf{k})$ for the individual magnon bands as ${ }^{33,34}$,

$$
\kappa_{x y}(T)=-\frac{k_{B}^{2} T}{4 \pi^{2} \hbar} \sum_{n= \pm} \iint_{B Z} c\left(\rho_{n}(\mathbf{k})\right) F_{n}(\mathbf{k}) d k_{x} d k_{y} .
$$

Here $T$ is the temperature, $k_{B}$ is the Boltzmann constant and $\hbar$ is the reduced Planck's constant. $F_{n}(\mathbf{k})$ is the Berry curvature of the $n$-th band. $c(x)=$ $(1+x)\left(\ln \frac{1+x}{x}\right)^{2}-(\ln x)^{2}-2 \operatorname{Li}_{2}(-x)$, where $\operatorname{Li}_{2}(z)=$ $-\int_{0}^{z} d u \frac{\ln (1-u)}{u}$ and $\rho_{n}(\mathbf{k})$ is the Bose-Einstein distribution, i.e., $\rho_{n}(\mathbf{k})=1 /\left(e^{E_{\mathbf{k}}^{n} / k_{B} T}-1\right) . \quad \kappa_{x y}(T)$ gets saturated at high temperatures like other thermodynamic quantities. The variation of $\kappa_{x y}(T)$ with temperature will be different for different topological phases since it directly depends on the Berry curvatures which actually determine the value of Chern numbers as well. Variation of $\kappa_{x y}$ with respect to $T$ for six different TMI phases is shown in Fig 8 with different colors. Saturated value of $\kappa_{x y}$ is positive (negative) when the sign of $C$ for upper band is negative (positive). On the other hand, absolute value of $\kappa_{x y}$ is found proportional to the value of $C$.

Also, transition among the various topological phases can be identified by studying the variations of $\kappa_{x y}$ in the parameter space for fixed value of $T$. There is a discontinuity in $\kappa_{x y}$ when the system crosses topological phase boundary. TPT of the system with respect to Kitaev terms $K^{\prime}$ and $K$ are shown in Figs 9 (a) and (b), respectively. In each case, the system undergoes two distinct transitions separating two different TMI phases by an intermediate trivial phase $C=0$. The system remains trivial at the transition points since no band gap is found to appear there. Similar kind of transitions can be observed by varying the other parameters as well. For the characterization of TMI phase for $\alpha-\mathrm{RuCl}_{3}$, values of the parameters in unit of $\mathrm{meV}$ are given, $J_{1}=-1.8$, $K_{1}=-10.6, J_{3}=1.25, K_{3}=0.65^{23}$. This material is topologically identified with the phase $C=\mp 1$. Similarly, $\mathrm{Li}_{2} \mathrm{IrO}_{3}\left(J_{1}=-3.0, K_{1}=-8.0, J_{3}=6.0\right)^{24}$, 


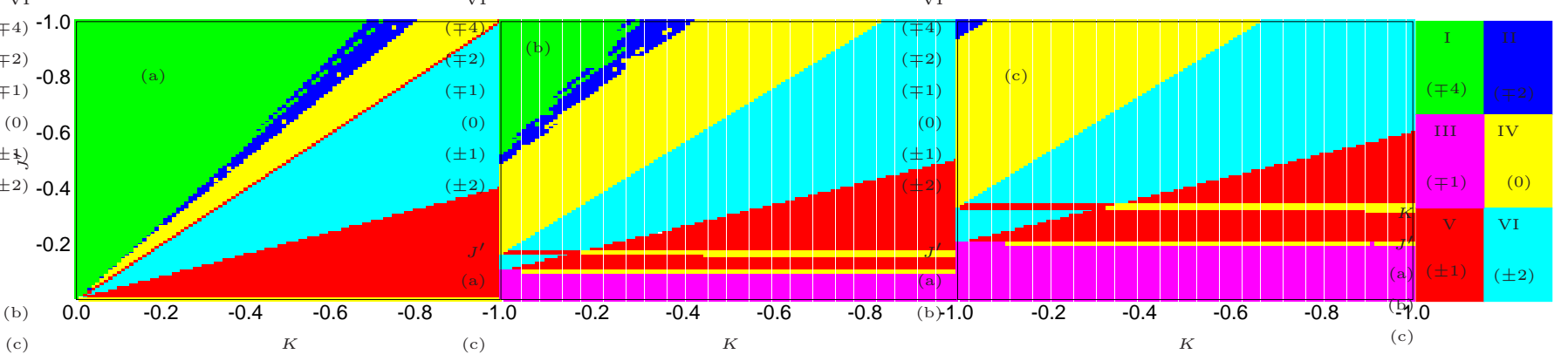

FIG. 6: (color online) Regions of TMI phases of the system in $J^{\prime}-K$ parameter space for (a) $J=0,(\mathrm{~b}) J=-0.5,(\mathrm{c}) J=-1$.

$\mathrm{Na}_{2} \mathrm{IrO}_{3}\left(J_{1}=3.6, K_{1}=-18.0, J_{3}=1.8\right)^{35}$, and $\mathrm{CrI}_{3}$ $\left(J_{1}=-0.2, K_{1}=-5.2\right)^{27}$ topologically belong to the same phase under external magnetic field.

\section{DISCUSSION}

This investigation reveals the emergence of multiple topological phases in Kitaev-Heisenberg ferromagnetic model on honeycomb lattice in the presence of both symmetric spin-anisotropic interaction and external magnetic field. The NN KHSA model exhibits a single topological phase $(C= \pm 1)$ when $\boldsymbol{h}$ acts along the $z$-axis. Another phase with $C=\mp 1$ appears if $\boldsymbol{h}$ points towards [111] direction $^{21}$. Here, it has been shown that in total six different topological phases $(C= \pm 1, \mp 1, \pm 2, \mp 2, \pm 3, \mp 4)$ appear with the inclusion of NNNN terms when $\boldsymbol{h}$ is directed along the $z$-axis. Therefore, a single FM ground state hosts six different TMI phases on the basis of magnon excitations. Whereas, no additional phase emerges when $\boldsymbol{h}$ points towards [111] direction. Again in contrast, no additional phase appears in the FM ordered regime under any condition with the inclusion of NNN terms. FM ground state bears the $U(1)$ rotational symmetry around the $z$ direction, although the Hamiltonian, Eq 1, does not comply with this symmetry. Analytic expressions of magnon dispersion relations are obtained following the LSWT based on this FM ground state. Thus, as far as the single magnon mode is concerned, the disper-

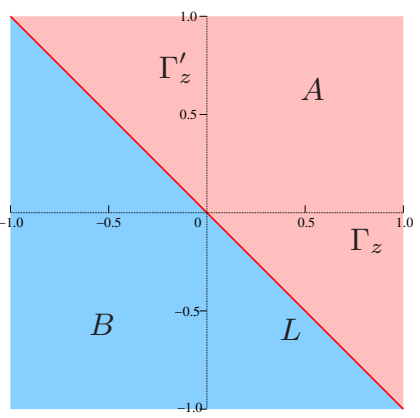

FIG. 7: (color online) Topological phase diagram of the system in $\Gamma_{z}-\Gamma_{z}^{\prime}$ space.

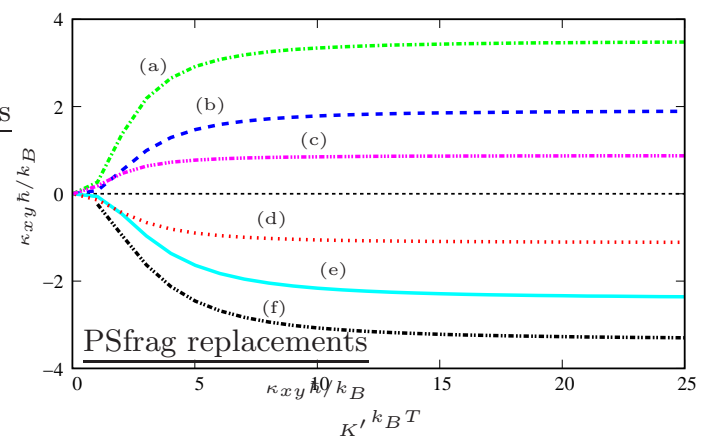

FIG. 8: (color online) Variation of $\kappa_{x y}(T)$ with $T$ for (a) $J=-0.5, J^{\prime}=-1, K^{\prime}=-1$, for $C=\mp 4$, (b) $J=-1$, $J^{\prime}=-1, K^{\prime}=-1$, for $C=\mp 2$, (c) $J=-1, K=-1$, for $C=\mp 1$, (d) $J=-1, K=-1, K^{\prime}=-1$, for $C= \pm 1,(\mathrm{e})$ $J=-1, K=-1, J^{\prime}=-1, K^{\prime}=-1$, for $C= \pm 2$, (f) $J=-2$, $K=-0.2, J^{\prime}=-1, K^{\prime}=0.4$ for $C= \pm 3$, with $\Gamma_{z}^{\prime}=0.5$ and $h=1$. No value is specified for those parameters when they are zero.
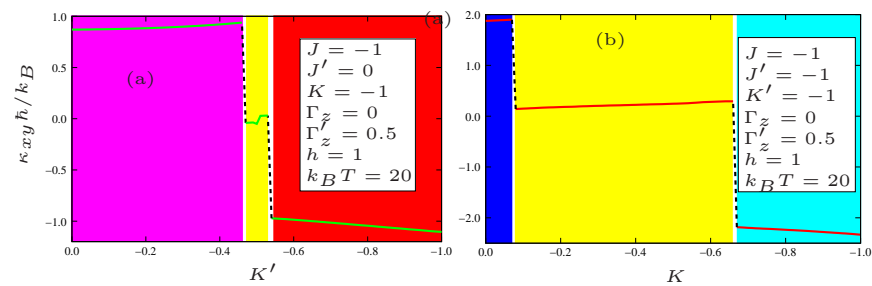

FIG. 9: (color online) Variation of $\kappa_{x y}$ in the parameter space when $T$ is fixed. Different regions are identified with distinct colors, those used before in Fig 6.

sion relations are exact and valid for any value of $S$. However, in this analysis, contribution of multi-magnon states is not taken into account, since, in a previous study, stability of those TMI phases based on the LSWT is found robust against the magnon-magnon interactions. Contribution of magnon-magnon interactions in the KHSA Hamiltonian has been treated before (i) perturbatively, (ii) nonperturbatively using density matrix renormalization group and (iii) numerically using exact diagonization of the Hamiltonian on a 24-site cluster ${ }^{20}$. It has been shown that topologically protected edge states obtained in LSWT are robust enough to withstand the presence 
of magnon-magnon interactions.

Exhaustive topological phase diagrams of this model are presented. Six different TMI phases are also characterized in terms of thermal behavior of Hall conductivity. TMI phase with the highest value of Chern number, $C=\mp 4$ dominates when $J=0$, but begins to disappear with the decrease of $J$ and ultimately vanishes at $J=-1$, when $J^{\prime}=K^{\prime}$. On the other hand, TMI phase with $C= \pm 3$ appears under various conditions. TPT driven by the parameters is also studied. Kitaev materials are found to belong into a definite TMI phase with
$C=\mp 1$. Experimental determination of THC for the Kitaev materials thus provides a natural way for the verification of their topological phases.

\section{ACKNOWLEDGMENTS}

MD acknowledges the UGC fellowship, no. 524067 (2014), India. AKG acknowledges BRNS-sanctioned research project, no. 37(3)/14/16/2015, India.
* Electronic address: moumitadeb44@gmail.com

$\dagger$ Electronic address: asimkumar96@yahoo.com

1 M Z Hasan and C L Kane, Rev. Mod. Phys. 82, 3045 (2010).

2 D J Thouless, M Kohomoto, P Nightingale and M den Nijs, Phys. Rev. Lett. 49, 405 (1982).

3 Y Hatsugai, Phys. Rev. Lett. 71, 3697 (1993).

4 Y Hatsugai, Phys. Rev. B 48, 11851 (1993).

${ }^{5}$ L Trifunovic and P W Brouwer, Phys. Rev. X 9, 011012 (2019).

6 Y Onose, T Ideue, H Katsura, Y Shiomi, N Nagaosa and Y Tokura, Science, 329, 297 (2010).

7 H Katsura, N Nagaosa and P A Lee, Phys. Rev. Lett. 104, 066403 (2010).

8 R Chisnell et. al., Phys. Rev. Lett. 115, 147201 (2015).

9 I Dzyaloshinskii, J. Phys. Chem. Solids 4, 241 (1958).

10 T Moriya, Phys. Rev. 120, 91 (1960).

11 L Zhang, J Ren, J-S Wang and B Li, Phys. Rev. B 87, 144101 (2013).

12 R Seshadri and D Sen, Phys. Rev. B 97, 134411 (2018).

13 S K Kim, H Ochoa, R Zaruela and Y Tserkovnyak, Phys. Rev. Lett. 117, 227201 (2016).

14 S A Owerre, J. Phys.: Condens. Matter 28, 386001 (2016).

15 J Romhányi, K Penc and R Ganesh, Nat. Commun. 6, 6805 (2015).

16 M Malki and K P Schmidt, Phys. Rev. B 95, 195137 (2017).

17 D Sticlet and F Piéchon, Phys. Rev. B 87, 115402 (2013).

18 A Sil and A K Ghosh, J. Phys.: Condens. Matter 31,
245601 (2019).

19 A Kitaev, Ann. Phys. 321, 2 (2006).

20 P A McClarty et. al., Phys. Rev. B 98, 060404(R) (2018).

21 D G Joshi, Phys. Rev. B 98, 060405(R) (2018).

22 H-S Kim, V Shankar V, A Catuneanu and H-Y Kee, Phys. Rev. B 91, 241110(R) (2015).

23 Y S Hou, H J Xiang and X G Gong, Phys. Rev. B 96, 054410 (2017).

24 S M Winter, Y li, H O Jeschke and R Valentí, Phys. Rev. B 93, 214431 (2016).

${ }^{25}$ Y Kasahara et. al., Nature 559, 227 (2018).

${ }^{26}$ V M Katukuri et. al., New J. Phys. 16, 013056 (2014).

27 I Lee et. al., arXiv:1902.00077 (2019).

28 J Chaloupka, G Jackeli and G Khaliullin, Phys. Rev. Lett. 110, 097204 (2013).

29 H-C Jiang, Z-C Gu, X-L Qi and S Trebst, Phys. Rev. B 83, 245104 (2011).

${ }^{30}$ L Janssen, E C Andrade and M Vojta, Phys. Rev. Lett. 117, 277202 (2016).

31 J G Rau, E K-H Lee and H-Y Kee, Phys. Rev. Lett. 112, 077204 (2014).

32 T Fukui, Y Hatsugai and H Suzuki, J. Phys. Soc. Jpn. 74, 1674 (2005).

33 R Matsumoto and S Murakami, Phys. Rev. Lett. 106, 197202 (2011).

34 R Matsumoto and S Murakami, Phys. Rev. B 84, 184406 (2011).

35 S D Das et. al., Phys. Rev. B 99, 081101(R) (2019). 